\title{
Gene expression analysis reveals marked differences in the transcriptome of infantile hemangioma endothelial cells compared to normal dermal microvascular endothelial cells
}

\author{
Vascular Cell 5:6 I DOI: 10.1186/2045-824X-5-6 I C Li et al.; licensee Publiverse Online S.R.L. 2013 \\ Received: 24 Jan 2013 | Accepted: 13 Jan 2013 | Published: 25 Jan 2013 \\ Stiles Jessica M, Rowntree Rebecca K, Amaya Clarissa, Diaz Dolores, Kokta Victor, Mitchell Dianne C, \\ Bryan Brad A \\ ${ }^{+}$Contributed equally ${ }^{\circledR}$ Corresponding author
}

\begin{abstract}
Background

Infantile hemangiomas are benign vascular tumors primarily found on the skin in $10 \%$ of the pediatric population. The etiology of this disease is largely unknown and while large scale genomic studies have examined the transcriptomes of infantile hemangioma tumors as a whole, no study to date has compared the global gene expression profiles of pure infantile hemangioma endothelial cells (HEMECs) to that of normal human dermal microvascular endothelial cells (HDMVECs).
\end{abstract}

\section{Methods}

To shed light on the molecular differences between these normal and aberrant dermal endothelial cell types, we performed whole genome microarray analysis on purified cultures of HEMECs and HDMVECs. We then utilized qPCR and immunohistochemistry to confirm our microarray results.

\section{Results}

Our array analysis identified 125 genes whose expression was upregulated and 104 genes whose expression was downregulated by greater than two fold in HEMECs compared to HDMVECs. Bioinformatics analysis revealed three major classifications of gene functions that were altered in HEMECs including cell adhesion, cell cycle, and arachidonic acid production. Several of these genes have been reported to be critical regulators and/or mutated in cancer, vascular tumors, and vascular malformations. We confirmed the expression of a subset of these differentially expressed genes (ANGPT2, ANTXR1, SMARCE1, RGS5, CTAG2, LTBP2, CLDN11, and KISS1) using qPCR and utilized immunohistochemistry on a panel of paraffin embedded infantile hemangioma tumor tissues to demonstrate that the cancer/testis antig en CTAG2 is highly abundant in vessel-dense proliferating infantile hemangiomas and with significantly reduced levels during tumor involution as vascular density decreases.

\section{Conclusion}

Our data reveal that the transcriptome of HEMECs is reflective of a pro-proliferative cell type with altered adhesive characteristics. Moveover, HEMECs show altered expression of many genes that are important in the progression and prognosis of metastatic cancers.

\section{Introduction}

Infantile hemangiomas are benign tumors of vascular origin that affect approximately $10 \%$ of the pediatric population. These tumors are characterized by a rapid proliferation phase over the first $1-2$ years of the child's life, followed by a slow and steady decline over the next 5-7 years leading to the complete involution of the tumor mass. Approximately $90 \%$ of all infantile hemangiomas remain small and are best left alone to naturally involute. However in about $10 \%$ of the cases the tumors exhibit aggressive characteristics based on their size, location, number, etc. and must be actively treated to avoid patient disfigurement and/or mortality.

The etiology of infantile hemangiomas is largely unknown, particularly with regard to the cellular origin of the tumor. Circumstantial evidence suggests that these lesions are of aberrant placental origin as evidenced by upregulated Glut1 expression [ 1], and some labs have ventured to hypothesize that they may be formed from metastatic invasion of placentaderived chorangioma cells [ 2]. Indeed, transcriptional profiling of human placenta, infantile hemangioma, and eight normal 
and diseased vascularized tissues suggests that high transcriptome similarity is shared between placenta and hemangioma tissues, more so than any of the other tissues tested [3]. Global gene expression analysis of infantile hemangioma tumors has been previously performed by two labs. Ritter et al. [4] utilized microarray analysis on whole tumors and identified immune regulators and indoleamine 2,3 dioxygenase as key regulators of infantile hemangioma involution. Calicchio et al. [ 5] utilized laser capture microdissection and genome-wide transcriptional profiling of vessels from proliferating and involuting hemangiomas. The authors strongly associated proliferating hemangioma vessels with increased expression of genes involved in endothelial-pericyte interactions and neuronal/vascular patterning, and involuting hemangiomas with chronic inflammatory mediators and angiogenic inhibitors. Given the high density of tightly associated pericytes in infantile hemangiomas and the inevitable collateral capture of intraluminal white cells, fibroblasts, mast cells, and perivascular collagen with laser microdissection, these data represent changes from numerous cell types within the infantile hemangioma tumor, but are not reflective specifically of the aberrant endothelial cells which contribute to disease. While these genomics studies have provided great mechanistic insight into the etiology and progression of the disease, they have not addressed the unique differences between abnormal infantile hemangioma endothelial cells and the normal dermal endothelial cells that are resident in the surrounding skin area of the patient. Understanding these differences could identify targetable pathways that could be exploited to preferentially block hemangioma growth and spread, but spare normal endothelial cells.

To date, no direct whole genome comparison of pure cultures of human dermal microvascular endothelial cells (HDMVECs) and infantile hemangioma endothelial cells (HEMECs) has been reported. To address this, we performed whole genome microarray profiling of the gene expression alterations between low passage pure cultures of HEMECs and HDMVECs. We identified a number of transcriptional alterations that are likely to contribute to the aggressive phenotype of infantile hemangiomas and that could potentially be utilized in immunotherapy against particularly aggressive hemangiomas tumors.

\section{Materials and methods}

\section{Cell culture and chemicals}

The HEMEC cell line was previously isolated from a proliferating-phase infantile hemangioma specimen collected from a female infant and generously donated to us by Joyce Bischoff (Harvard Medical School) [ 6]. The primary culture of neonatal HDMVECs was purchased from ATCC. Both cell lines were cultured as previously reported [ 7]. For all experiments, cell lines were used at $<5$ passages.

\section{Proliferation assay}

Cells were plated at equivalent sub-confluent densities and maintained in a Nikon Biostation CT time lapse imaging station. Cell proliferation was measured by counting cells per vision field from 5 independent areas over a 96 hour time course. Data presented is the average of the counts plus or minus the standard deviation. Student's $t$-test was used to evaluate statistical significance. Data with $\mathrm{p}<0.05$ was considered significant.

\section{Migration assay}

Confluent cultures were scratch wounded and the progress of "wound healing" was monitored using a Nikon Biostation CT time lapse imaging station over a 9 hour period. Data presented is the average migration speed plus or minus the standard deviation. Student's $t$-test was used to evaluate statistical significance $(\mathrm{p}<0.05)$. Data with $\mathrm{p}<0.05$ was considered significant.

\section{Immunofluorescence}

Cells were plated onto collagen type I coated glass coverslips, fixed in $4 \%$ paraformaldehyde, and incubated with antibodies against phospho-focal adhesion kinase (p-FAK; 1:1000; Cell Signaling \#3283), rhodamine conjugated phalloidin (1:350; Cytoskeleton Inc.), or DAPI and imaged via a Nikon Eclipse Ti laser scanning confocal microscope.

\section{Microarray analysis}

Total RNA was amplified and biotin-labeled using Illumina TotalPrep RNA Amplification Kit (Ambion). $750 \mathrm{ng}$ of biotinylated aRNA was then briefly heat-denatured and loaded onto expression arrays to hybridize overnight. Following hybridization, arrays were labeled with $\mathrm{Cy} 3$-streptavidin and imaged on the Illumina ISCAN. Intensity values were transferred to Agilent GeneSpring GX microarray analysis software and data was filtered based on quality of each call. Statistical relevance was determined using ANOVA with a Benjamini Hochberg FDR multiple testing correction ( $p$-value < 0.05). Data were then limited by fold change analysis to statistically relevant data points demonstrating a 2-fold or more change in expression. Pathway analysis was performed using Metacore software. The microarray data from this experiment is publically available on the Gene Expression Omnibus (GEO Accession \#GSE43742).

\section{Quantitative real time PCR analysis}

RNA was isolated from cells using the Ambion Purelink Minikit according to the manufacturer's directions. qRT-PCR was performed on an ABI7900HT RT-PCR system using TaqMan Assays with predesigned primer sets for the genes of interest (Invitrogen). All RT-PCR experiments were performed in triplicate. 
Paraffinized infantile hemangioma tissues were labeled with CTAG2 antibody (1:200, Santa Cruz Biotechnology \#sc99243) and quantified using Alkaline Phosphatase detection (CellMarque). Positive and negative controls from breast carcinoma tissues were stained with CTAG2 antibody or sham, respectively. Use of de-identified human tissues was approved by the Texas Tech University Health Sciences Center Institutional Review Board for the Protection of Human Subjects (IRB E13029). Waiver of informed consent was approved by IRB.

\section{Results and discussion}

A comparison of the proliferation and migration rates of HEMECs and HDMVECs under standard growth conditions revealed no significant difference between normal and hemangioma endothelial cell types, however HEMECs grown under reduced serum conditions ( $0.5 \%$ fetal bovine serum) exhibited an approximately $30 \%$ increase in proliferation and an approximately $18 \%$ increase in migration relative to HDMVECs grown under the same conditions (Figure $1 \mathrm{~A} \& \mathrm{~B}$ ). This suggests the higher serum concentrations were likely masking any phenotypic advantage attributed to the HEMECs. Moreover, it indicates the proliferative and migratory capacity of HEMECs are unique from that observed in HDMVECs and agrees with earlier reports suggesting advantages in these areas for HEMECs [6]. Comparisons of fluorescent images of the actin cytoskeleton and active focal adhesion complexes obtained with confocal microscopy revealed that HDMVECs display primarily peripheral membrane localized p-FAK, indicating sites of cellular attachment to the extracellular matrix (ECM) (Figure 1C). In contrast, p-FAK localization in HEMECs was observed along the entirety of the actin stress fibers, suggesting cellular adhesion to its substrate is markedly altered in HEMECs. Indeed, it has previously been reported that HEMECs display unique expression of genes involved in cellular adhesion [ 8]. Figure 1

Analysis of HDMVEC and HEMEC phenotypes.( A) Analysis of proliferation rates between HDMVECs and HEMECs over a $48 \mathrm{hr}$ time course. ( B) Analysis of the migration rates of HDMVECs and HEMECs nine hours after initial scratch from a micropipette. ( C) Immunofluorescent imaging of actin ( red ), p-FAK ( green ), and nucleus ( blue ). (red asterisks for panels A\& Brepresent statistically significant values $[\mathrm{p}<0.05]$ as determined by Student's $t$-test).

\section{Whole genome microarray analysis reveals large scale alterations in gene expression between HEMECs and HDMVECs}

Given the phenotypic differences observed between HEMECs and HDMVECs, we compared the global gene expression patterns between pure cultures of these cells using Illumina high density BeadArrays to elucidate which molecular factors are deregulated in HEMECs. Our array analysis identified 125 genes whose expression was upregulated and 104 genes whose expression was downregulated ( 2 fold or greater, $\mathrm{p}<0.05$ ) in HEMECs compared to HDMVECs (Table 1 ). Metacore analysis of the 2 fold or greater gene expression changes revealed three major classifications of gene functions that are altered in HEMECs including cell adhesion ( TIMP1, COL1A1, COL1A2, MMP1, MMP13, SERPINE2, COLAA6, LAMC2, MMP2, CD44, CAV1, CCL2, JAM3, CLDN11, LYVE1 ), cell cycle ( CCND2, CDKN2A, CCNA1, NCAPD2 ), and arachidonic acid production ( $A C S L 5, F A P, L I P G, P L A 2 G 4 C$ ). Given the number of adhesion genes whose expression is altered in HEMECs compared to HDMVECs, it is no surprise that we observed altered subcellular localization of p-FAK in HEMECs (Figure 1C), reflecting a unique adhesive phenotype in these cells. Our data reflect altered cell cycle regulation in HEMECs, with a downregulation of CCND2 ( cyclin D2) and CDKN2A (p16Ink4A) and a potent 6.6 fold increase in CCNA1 (cyclin A1), and these changes may contribute to the enhanced proliferation rates in HEMECs and the uncontrolled cell growth observed in infantile hemangiomas tumors. Alterations in the expression of genes involved in arachidonic acid production were unique in that this polyunsaturated fatty acid can serve as a lipid second messenger in the regulation of phospholipase- $\mathrm{C}$ and protein kinase-C signaling, is a key inflammatory intermediate, and can act as a vasodilator [9].

Table 1

\begin{tabular}{|c|c|c|c|}
\hline Gene symbol & Gene name & Accession number & FC \\
\hline CTAG2 & Cancer/testis antigen 2 & NM_020994.3 & 11.6 \\
\hline IL13RA2 & Interleukin 13 Receptor, alpha 2 & NM_000640.2 & 10.7 \\
\hline IFI27 & Interferon, alpha-inducible protein 27 & NM_005532.3 & 8.3 \\
\hline TPM2 & Tropomyosin 2 (beta) & NM_213674.1 & 7.8 \\
\hline RPL14 & Ribosomal protein L14 & NM_001034996.1 & 6.6 \\
\hline CCNA1 & Cyclin A1 & NM_003914.3 & 6.6 \\
\hline RGS5 & G-protein signaling 5 regulator & NM_003617.3 & 6.0 \\
\hline FBN2 & Fibrillin 2 & NM_001999.3 & 5.9 \\
\hline D4S234E & DNA segment on chromosome 4 (unique) & NM_001040101.1 & 5.5 \\
\hline BST2 & Bone marrow stromal cell antigen 2 & NM_004335.2 & 5.1 \\
\hline QPCT & Glutaminyl-peptide cyclotransferase & NM_012413.3 & 4.8 \\
\hline TNFSF4 & Tumor necrosis factor (ligand) superfamily, member 4 & NM_003326.3 & 4.6 \\
\hline RGS5 & Regulator of G-protein signaling 5 & NM_003617.3 & 4.6 \\
\hline SPOCK 1 & Sparc/osteonectin, cwcv and kazal-like domains proteoglycan 1 & NM_004598.3 & 4.6 \\
\hline SNHG8 & Small nucleolar RNA host gene 8 (non-protein coding) & NR_003584.3 & 4.6 \\
\hline ANTXR1 & Anthrax toxin receptor 1 & NM_032208.2 & 4.5 \\
\hline CHST1 & Carbohydrate sulfotransferase 1 & NM_003654.5 & 4.5 \\
\hline
\end{tabular}




\begin{tabular}{|c|c|c|c|}
\hline Gene symbol & Gene name & Accession number & FC \\
\hline MPZL2 & Myelin protein zero-like 2 & NM_005797.3 & 4.4 \\
\hline HEY2 & Hairy/enhancer-of-spilt related with YRPW motif 2 & NM_012259.2 & 4.3 \\
\hline SLITRK4 & SLIT and NTRK-like family, member 4 & NM_173078.3 & 4.2 \\
\hline SHISA2 & Shisa homolog 2 & NM_001007538.1 & 4.0 \\
\hline LRRC17 & Leucine rich repeat containing 17 , TV2 & NM_005824.2 & 3.9 \\
\hline NUDT11 & Nudix-type motif 11 & NM_018159.3 & 3.8 \\
\hline RNASE1 & Ribonuclease, Rnase A family, 1, TV1 & NM_198235.2 & 3.7 \\
\hline SERPINE2 & Serpin peptidase inhibitor, clade E, member 2 & NM_006216.3 & 3.6 \\
\hline LIPG & Lipase, endothelial & NM_006033.2 & 3.4 \\
\hline PCSK5 & Proprotein convertase subtilisin/kexin type 5 & NM_006200.3 & 3.4 \\
\hline LPXN & Leupaxin & NM_004811.2 & 3.3 \\
\hline CXCR4 & Chmeokine (C-X-C motif) receptor 4 , TV2 & NM_003467.2 & 3.2 \\
\hline TMEM200A & Transmembrane protein $200 \mathrm{~A}$ & NM_052913.2 & 3.1 \\
\hline CXCR4 & Chemokine (C-X-C motif) receptor 4, TV1 & NM_001008540.1 & 3.1 \\
\hline RAB34 & RAB34, member RAS onogene family & NM_031934.5 & 3.0 \\
\hline DPYSL3 & Dihydropyrimidinase-like 3 & NM_001387.2 & 2.9 \\
\hline FBXL13 & F-box and leucine-rich repeat protein 13 & NM_145032.3 & 2.9 \\
\hline PNMA2 & Paraneoplastic Ma antigen 2 & NM_007257.5 & 2.9 \\
\hline LOC440354 & LOC440354 & NR_002473.2 & 2.9 \\
\hline NLGN1 & Neuroligin 1 & NM_014932.2 & 2.8 \\
\hline DDIT4 & DNA-damage-inducible transcript 4 & NM_019058.2 & 2.8 \\
\hline PFN2 & Profilin 2 & NM_053024.3 & 2.8 \\
\hline GABBR2 & Gamma-aminobutyric acid B receptor, 2 & NM_005458.7 & 2.8 \\
\hline MEIS2 & Meis homeobox 2 & NM_172315.2 & 2.7 \\
\hline PMEPA1 & Prostate transmembrane protein, androgen induced 1 & NM_199169.2 & 2.7 \\
\hline LOC647307 & LOC647308 & XR_039752.1 & 2.7 \\
\hline PLEK2 & Pleckstrin 2 & NM_016445.1 & 2.7 \\
\hline CARD11 & Caspase recruitment domain family, member 11 & NM_032415.4 & 2.6 \\
\hline SNORD13 & Small nucleolar RNA, C/D box 13, small nucleolar RNA & NR_003041.1 & 2.6 \\
\hline GFPT2 & Glutamine-fructoce-6-phosphate transaminase 2 & NM_005110.2 & 2.6 \\
\hline FAP & Fibroblast activation protein, alpha & NM_004460.2 & 2.6 \\
\hline OCIAD2 & OCIA domain containing $2, \mathrm{TV} 2$ & NM_152398.2 & 2.5 \\
\hline F2RL1 & Coagulation factor II receptor-like 1 & NM_005242.4 & 2.5 \\
\hline DSTYK & Dual serine/threonine and tyrosine protein kinase & NM_199462.2 & 2.5 \\
\hline LOC649497 & LOC649498 & XM_938576.1 & 2.5 \\
\hline LOC654194 & LOC654195 & XM_942669.1 & 2.5 \\
\hline NYNRIN & NYN domain and retroviral integrase containing & NM_025081.2 & 2.5 \\
\hline LOC387763 & LOC387764 & XM_941665.2 & 2.5 \\
\hline COL8A1 & Collagen, type VIII, alpha 1 & NM_020351.3 & 2.5 \\
\hline MGC39900 & MGC39901 & XM_936687.1 & 2.4 \\
\hline LTBP2 & Latent transforming growth factor beta binding protein 2 & NM_000428.2 & 2.4 \\
\hline RNASE1 & Ribonuclease, Rnase A family, 1, TV3 & NM_198232.2 & 2.4 \\
\hline IFI27L2 & Interferon, alpha-inducible protein 27 -like 2 & NM_032036.2 & 2.4 \\
\hline SOX4 & SRY (sex determining region Y)-box4 & NM_003107.2 & 2.4 \\
\hline LRRC17 & Leucine rich repeat containing $17, \mathrm{TV} 1$ & NM_001031692.2 & 2.3 \\
\hline DSE & Dermatan sulfate epimerase & NM_013352.2 & 2.3 \\
\hline CD44 & CD44 molecule (Indian blood group), TV5 & NM_001001392.1 & 2.3 \\
\hline LOC100131139 & LOC100131140 & XR_037336.1 & 2.3 \\
\hline CBS & Systathionine-beta-synthase & NM_000071.2 & 2.3 \\
\hline NT5DC2 & 5 '-nucleotidase domain containing 2 & NM_022908.2 & 2.3 \\
\hline NPFFR2 & Neuropeptide FF receptor 2 & NM_004885.2 & 2.3 \\
\hline LOC100129685 & LOC100129686 & XM_001723814.1 & 2.3 \\
\hline LXN & Latexin & NM_020169.3 & 2.3 \\
\hline MEX3B & Mex-3 homolog B & NM_032246.3 & 2.3 \\
\hline C1orf54 & Chromosome 1 open reading frame 54 & NM_024579.3 & 2.3 \\
\hline HDDC2 & HD domain containing 2 & NM_016063.2 & 2.3 \\
\hline LOC648823 & LOC648824 & XM_943477.1 & 2.3 \\
\hline CYB5A & Cytochrome b5 type A & NM_001914.3 & 2.3 \\
\hline PIR & Pirin (iron binding nuclear protein) & NM_001018109.2 & 2.3 \\
\hline
\end{tabular}




\begin{tabular}{|c|c|c|c|}
\hline Gene symbol & Gene name & Accession number & FC \\
\hline GPR37 & G protein-coupled receptor 37 & NM_005302.2 & 2.3 \\
\hline PPAPDC1A & Phosphatidic acid phosphatase type 2 domain containing $1 \mathrm{~A}$ & NM_001030059.1 & 2.3 \\
\hline CD44 & CD44 molecule (Indian blood group), TV4 & NM_001001391.1 & 2.2 \\
\hline LOC100131905 & LOC100131906 & XR_039334.1 & 2.2 \\
\hline CTAG1A & Cancer/testis antigen $1 \mathrm{~A}$ & NM_139250.1 & 2.2 \\
\hline C4orf18 & Chromosome 4 open reading frame 18 & NM_016613.6 & 2.2 \\
\hline LDOC1 & Leucine zipper, down-regulated in cancer 1 & NM_012317.2 & 2.2 \\
\hline TGFBI & Transforming growth factor, beta-induced & NM_000358.2 & 2.2 \\
\hline COL5A2 & Collagen, type $\mathrm{V}$, alpha 2 & NM_000393.3 & 2.2 \\
\hline NOX4 & NADPH oxidase 4 & NM_016931.3 & 2.2 \\
\hline TSHZ3 & Teashirt zinc finger homeobox 3 & NM_020856.2 & 2.2 \\
\hline FNDC3B & Fibronectin type III domain containing 3B, TV2 & NM_001135095.1 & 2.2 \\
\hline KIT & V-kit & NM_001093772.1 & 2.2 \\
\hline ADAM19 & ADAM metallopeptidase domain 19 & NM_033274.3 & 2.2 \\
\hline JAM3 & Junctional adhesion molecule 3 & NM_032801.4 & 2.1 \\
\hline CGNL1 & Cingulin-like 1 & NM_032866.4 & 2.1 \\
\hline COL4A6 & Collagen, type IV, alpha 6 & NM_001847.2 & 2.1 \\
\hline BMX & BMX non-receptor tyrosine kinase & NM_001721.6 & 2.1 \\
\hline DUSP23 & Dual specificity phosphatase 23 & NM_017823.3 & 2.1 \\
\hline MMP2 & Matrix metallopeptidase 2 & NM_004530.4 & 2.1 \\
\hline NCAPD2 & Non-SMC condensin I complex, subunit D2 & NM_014865.3 & 2.1 \\
\hline CYBRD1 & Cytochrome $\mathrm{b}$ reductase $1, \mathrm{TV} 1$ & NM_024843.2 & 2.1 \\
\hline FAM89A & Family with sequence similarity 89 , member A & NM_198552.2 & 2.1 \\
\hline GAS6 & Growth arrest-specific 6 & NM_000820.2 & 2.1 \\
\hline S100A13 & S100 calcium binding protein $\mathrm{A} 13$ & NM_001024211.1 & 2.1 \\
\hline SMARCE1 & SWI/SNF related, subfamily e, member 1 & NM_003079.4 & 2.1 \\
\hline LOC643977 & LOC643978 & XM_932991.1 & 2.1 \\
\hline LFNG & O-fucosylpeptide 3-beta- $\mathrm{N}$-acetylglucosaminyltransferase & NM_001040167.1 & 2.1 \\
\hline MTMR11 & Myotubularin related protein 11 & NM_181873.3 & 2.1 \\
\hline ITGA10 & Integrin, alpha 10 & NM_003637.3 & 2.1 \\
\hline PTGFRN & Prostaglandin F2 receptor negative regulator & NM_020440.2 & 2.0 \\
\hline LOC644936 & Actin, beta pseudogene & NR_004845.1 & 2.0 \\
\hline CPS1 & Carbamoyl-phosphate synthase 1 , mitochonfrial & NM_001875.4 & 2.0 \\
\hline C18orf56 & Chromosome 18 open reading frame 56 & NM_001012716.2 & 2.0 \\
\hline ADA & Adenosine deaminase & NM_000022.2 & 2.0 \\
\hline NETO2 & Neuropilin and tolliod-like2 & NM_018092.4 & 2.0 \\
\hline DKFZp761P0423 & DKFZp761P0424 & XM_291277.4 & 2.0 \\
\hline STC2 & Stanniocalcin 2 & NM_003714.2 & 2.0 \\
\hline PRKAR1A & Protein kinase, cAMP-dependent, regulatory, type I, alpha & NM_002734.3 & 2.0 \\
\hline EGFLAM & EGF-like, fibronectin type III and laminin G domains & NM_182801.2 & 2.0 \\
\hline SPECC1 & Sperm antigen with calponin homology, coiled-coil domains 1 & NM_001033555.2 & 2.0 \\
\hline FNDC3B & Fibronectin type III domain containing 3B, TV1 & NM_022763.3 & 2.0 \\
\hline THOC3 & THO complex 3 & NM_032361.2 & 2.0 \\
\hline COL5A1 & Collagen, type $\mathrm{V}$, alpha 1 & NM_000093.3 & 2.0 \\
\hline LANCL1 & LanC lantibiotic synthetase component C-like 1 & NM_006055.2 & 2.0 \\
\hline OCIAD2 & OCIA domain containing $2, \mathrm{TV} 1$ & NM_001014446.1 & 2.0 \\
\hline LRIG1 & Leucine-rich repeats and immunoglobulin-like domains 1 & NM_015541.2 & 2.0 \\
\hline HOXB2 & Homeobox B2 & NM_002145.3 & 2.0 \\
\hline TIMP1 & TIMP metallopeptidase inhibitor 1 & NM_003254.2 & -2.0 \\
\hline NAAA & $\mathrm{N}$-acylethanolamine acid amidase & NM_014435.3 & -2.0 \\
\hline MAOA & Monoamine oxidase A & NM_000240.2 & -2.0 \\
\hline MYOF & Myoferlin & NM_013451.3 & -2.0 \\
\hline KISS1 & KiSS metastasis-suppressor & NM_002256.3 & -2.0 \\
\hline SLC25A22 & Solute carrier family 25 , member 22 & NM_024698.5 & -2.0 \\
\hline NOSIP & Nitric oxide synthase interacting protein & NM_015953.3 & -2.0 \\
\hline COL1A2 & Collagen, type I, alpha 2 & NM_000089.3 & -2.0 \\
\hline ZDHHC14 & Zinc finger, DHHC-type containing 14 & NM_024630.2 & -2.0 \\
\hline HPCAL1 & Hippocalcin-like 1 & NM_134421.1 & -2.0 \\
\hline VLDLR & Very low density lipoprotein receptor & NM_001018056.1 & -2.0 \\
\hline
\end{tabular}




\begin{tabular}{|c|c|c|c|}
\hline Gene symbol & Gene name & Accession number & FC \\
\hline LOC730525 & LOC730525 & XM_001126202.1 & -2.0 \\
\hline BMP2 & Bone morphogenetic protein 2 & NM_001200.2 & -2.0 \\
\hline ABLIM1 & Actin binding LIM protein 1 & NM_006720.3 & -2.0 \\
\hline PIK3C2A & Phosphoinositide-3-kinase, class 2, alpha polypeptide & NM_002645.2 & -2.0 \\
\hline IRF1 & Interferon regulatory factor 1 & NM_002198.2 & -2.0 \\
\hline MBP & Myelin basic protein & NM_001025100.1 & -2.0 \\
\hline PRKAR1B & Protein kinase, cAMP-dependent, regulatory type I, beta & NM_002735.2 & -2.1 \\
\hline FAM101B & Family with sequence similarity 101 , member B & NM_182705.2 & -2.1 \\
\hline ERCC2 & DNA excision repair protein 2 & NM_000400.3 & -2.1 \\
\hline CCND2 & Cyclin D2 & NM_001759.3 & -2.1 \\
\hline HLA-B & Major histocompatibility complex, class I, B & NM_005514.6 & -2.1 \\
\hline SYBU & Syntabulin & NM_001099743.1 & -2.1 \\
\hline PDE2A & Phosphodiesterase 2A, cGMP-stimulated & NM_002599.4 & -2.1 \\
\hline AKAP12 & A kinase anchor protein 12 & NM_005100.3 & -2.1 \\
\hline CLEC2B & C-type lectin domain family 2 , member B & NM_005127.2 & -2.1 \\
\hline S100A4 & S100 calcuim bin ding protein A4 & NM_019554.2 & -2.1 \\
\hline FST & Follistain & NM_013409.2 & -2.2 \\
\hline SLC30A3 & Solute carrier family 30 , member 3 & NM_003459.4 & -2.2 \\
\hline PLIN2 & Perilipin 2 & NM_001122.3 & -2.2 \\
\hline IL32 & Interleukin 32 & NM_001012633.1 & -2.2 \\
\hline LOC100128252 & LOC100128253 & XM_001725603.1 & -2.2 \\
\hline TIMM22 & Translocase of inner mitochondrial membrane 22 homolog & NM_013337.2 & -2.2 \\
\hline SYNM & Synemin, intermediate filament protein & NM_015286.5 & -2.2 \\
\hline LOC729985 & LOC729986 & XM_001131964.1 & -2.2 \\
\hline ADRB2 & Adrenergic, beta-2-, receptor surface & NM_000024.5 & -2.2 \\
\hline KIAA1274 & KIAA1274 & NM_014431.2 & -2.2 \\
\hline PRR5 & Proline rich 5 & NM_001017529.2 & -2.2 \\
\hline LOC387841 & LOC387842 & XM_932678.1 & -2.3 \\
\hline CFI & Complement factor I & NM_000204.3 & -2.3 \\
\hline LOC646836 & LOC646837 & XM_001718162.1 & -2.3 \\
\hline COL1A1 & Collagen, type I, alpha 1 & NM_000088.3 & -2.3 \\
\hline CCL2 & Chemokine (C-C motif) ligand 2 & NM_002982.3 & -2.3 \\
\hline COL6A1 & Collagen, type VI, alpha 1 & NM_001848.2 & -2.3 \\
\hline LOC201651 & LOC201652 & XR_017321.2 & -2.3 \\
\hline GALNTL4 & GalNAc-T-like protein 4 & NM_198516.2 & -2.3 \\
\hline S100A3 & S100 calcuim binding protein A3 & NM_002960.1 & -2.4 \\
\hline ALDH1A1 & Aldehyde dehydrogenase 1 family, member A1 & NM_000689.4 & -2.4 \\
\hline TNFRSF14 & Tumor necosis factor receptor superfamily, member 14 & NM_003820.2 & -2.4 \\
\hline CAV1 & Caveolin 1 & NM_001753.4 & -2.4 \\
\hline LAMC2 & Laminin, gamma 2 & NM_005562.2 & -2.4 \\
\hline NOSTRIN & Nitric oxide synthase trafficker & NM_052946.3 & -2.4 \\
\hline CEACAM1 & Carcinoembryonic antigen-related cell adhesion molecule 1 & NM_001024912.2 & -2.4 \\
\hline CYYR1 & Cysteine/tyrosine-rich 1 & NM_052954.2 & -2.5 \\
\hline SLC22A23 & Solute carrier family 22 , member 23 & NM_021945.5 & -2.5 \\
\hline ACSL5 & Acyl-CoA synthetase long-chain family member 5 & NM_016234.3 & -2.5 \\
\hline AADAC & Arylacetamide deacetylase & NM_001086.2 & -2.6 \\
\hline COLEC12 & Collectin sub-family member 12 & NM_130386.2 & -2.6 \\
\hline KIAA1324L & KIAA1324-like & NM_152748.3 & -2.6 \\
\hline RNASET2 & Ribonuclease $\mathrm{T} 2$ & NM_003730.4 & -2.6 \\
\hline $\mathrm{NXN}$ & Nucleoredoxin & NM_022463.4 & -2.6 \\
\hline PLA2G4C & Phospholipase A2, group IVC & NM_003706.2 & -2.6 \\
\hline SERPINB2 & Serpin peptidase inhibitor, clade $B$, member 2 & NM_002575.2 & -2.6 \\
\hline CETP & Cholesteryl ester transfer protein, plasma & NM_000078.2 & -2.7 \\
\hline PLA2G16 & Phospholipase A2, group XVI & NM_007069.3 & -2.7 \\
\hline TNFSF18 & Tumor necrosis factor superfamily, member 18 & NM_005092.3 & -2.8 \\
\hline CITED2 & Cbp/p300-interacting transactivator 2 & NM_006079.3 & -2.8 \\
\hline C10orf116 & Chromosome 10 open reading fame 116 & NM_006829.2 & -2.8 \\
\hline PROX1 & Prospero homeobox 1 & NM_002763.3 & -2.9 \\
\hline PALM & Paralemmin & NM_002579.2 & -2.9 \\
\hline
\end{tabular}




\begin{tabular}{|c|c|c|c|}
\hline Gene symbol & Gene name & Accession number & FC \\
\hline ZSCAN18 & Zinc finger and SCAN domain containing 18 & NM_023926.4 & -2.9 \\
\hline LEPREL1 & Leprecan-like 1 & NM_018192.3 & -2.9 \\
\hline CTSH & Cathepsin H & NM_004390.3 & -2.9 \\
\hline KHDRBS3 & RNA-binding protein T-Star & NM_006558.1 & -3.0 \\
\hline CDH11 & Cadherin 11 , type 2 , OB-cadherin & NM_001797.2 & -3.1 \\
\hline DDIT4L & DNA-damage-inducible transcript 4-like & NM_145244.3 & -3.2 \\
\hline GAPDHL6 & GAPDHL7 & XM_001726954.1 & -3.2 \\
\hline NR5A2 & Nuclear receptor subfamily 5 , group A, member 2 & NM_003822.3 & -3.3 \\
\hline АBCA3 & ATP-binding cassette, sub-family A, member 3 & NM_001089.2 & -3.3 \\
\hline MARCH2 & Membrane-associated ring finger 2 & NM_001005416.1 & -3.3 \\
\hline CDKN2A & Cyclin-dependent kinase inhibitor $2 \mathrm{~A}$ & NM_000077.4 & -3.3 \\
\hline MGP & Matrix Gla protein & NM_000900.3 & -3.3 \\
\hline ALDH1A2 & Aldehyde dehydrogenase 1 family, member A2 & NM_170697.2 & -3.5 \\
\hline HOXB7 & Homeobox B7 & NM_004502.3 & -3.5 \\
\hline EMCN & Endomucin & NM_016242.3 & -3.5 \\
\hline ANGPT2 & Angiopoietin 2 & NM_001147.2 & -3.5 \\
\hline GIMAP5 & GTPase, IMAP family member 5 & NM_018384.4 & -3.6 \\
\hline NDN & Necdin homolog & NM_002487.2 & -3.8 \\
\hline TACSTD2 & Tumor associate calcuim signal transducer 2 & NM_002353.2 & -3.8 \\
\hline KRT19 & Keratin 19 & NM_002276.4 & -3.8 \\
\hline FAM174B & Family with sequence similarity 174 , member B & NM_207446.2 & -3.9 \\
\hline CECR1 & Cat eye syndrome chromosome region, candidate 1 & NM_177405.1 & -4.2 \\
\hline GPR116 & G protein-coupled receptor 116 & NM_015234.4 & -4.3 \\
\hline TNFRSF6B & Tumor necrosis factor superfamily, member $6 \mathrm{~b}$, decoy & NM_032945.2 & -4.3 \\
\hline PIEZO2 & Piezo-type mechanosensitive ion channel component 2 & NM_022068.2 & -4.4 \\
\hline UCHL1 & Ubiquitin carboxyl-terminal esterase L1 & NM_004181.4 & -4.9 \\
\hline KBTBD11 & Kelch repeat and BTB domain containing 11 & NM_014867.2 & -5.3 \\
\hline LOC375295 & LOC375296 & XM_374020.4 & -5.5 \\
\hline HSD17B2 & Hydroxysteroid dehydrogenase 2 & NM_002153.2 & -8.4 \\
\hline LYVE1 & Lymphatic vessel endothelial hyaluronan receptor 1 & NM_006691.3 & -8.8 \\
\hline PDPN & Podoplanin & NM_001006625.1 & -15.8 \\
\hline GYPC & Glycophorin C & NM_016815.3 & -22.6 \\
\hline MMP1 & Matrix metallopeptidase 1 & NM_002421.3 & -25.8 \\
\hline FABP4 & Fatty acid binding protein 4 , adipocyte & NM_001442.2 & -28.1 \\
\hline CLDN11 & Claudin 11 & NM_005602.5 & -36.9 \\
\hline
\end{tabular}

Fold changes in mRNA expression levels of genes in HEMECs compared to HDMVECs

We confirmed a small subset of these gene expression changes utilizing $\mathrm{qPCR}$, revealing equivocal trends in gene expression between the microarray and qPCR data for ANGPT2, ANTXR1, SMARCE1, RGS5, CTAG2, LTBP2, CLDN11, and KISSI (Table 2). Each of these genes has been firmly established to play critical roles in regulating angiogenesis and/or tumor progression [ 10-17]. Missense mutations in ANTXRI have been reported in several infantile hemangiomas and contribute to the constitutive VEGFR2 signaling associated with these tumors [ 18]. Mutations and signaling aberrations in Tie2, the cognate receptor for ANGPT2, play central roles in the development of various vascular disorders [ 19,20]. ANGPT2 has previously been shown to be down-regulated in response to serum in HEMECs [ 19]. Interestingly, ANGPT2 expression is higher in HEMECs compared to normal placental endothelial cells and is increased in proliferative infantile hemangioma tumors relative to involuting ones [ 5]. Virtually undetectable in normal vasculature, RGS5 is greatly upregulated in the vasculature of solid tumors and may have the potential to serve as a tumor biomarker [12]. The downregulation of the metastasis suppressor KISS1 that we observed in HEMECs may partially explain the locally aggressive properties of infantile hemangiomas, as this gene encodes an angiogenic suppressor [ 16, 21]. Moreover, the expression of KISSI is markedly reduced in aggressive metastatic melanomas and breast cancers, and this loss of expression contributes to the metastatic phenotype of these cells [ 17,22]. It is intriguing that such genes (particularly the cancerspecific genes) are aberrantly expressed in HEMECs, and undoubtedly their deregulation could potentiate aberrant vascular tumor states. As it has been proposed that infantile hemangiomas may be derived from motile placental-derived chorangioma cells [ 2], future genomics analysis should compare the transcriptomes of each tumor type to identify if aberrant expression of tumor-related genes is shared between the tissues.

\section{Table 2}

\begin{tabular}{l|l}
\hline \multicolumn{1}{c|}{ Gene } & Expression $\boldsymbol{\Delta}$ \\
\hline RGS5 & $92.4 \pm 11.2$ \\
CTAG2 & $39.9 \pm 4.8$ \\
SMARCE1 & $4.4 \pm 1.4$ \\
LTBP2 & $3.3 \pm 0.5$
\end{tabular}




\begin{tabular}{l|l}
\hline \multicolumn{1}{c|}{ Gene } & Expression $\boldsymbol{\Delta}$ \\
\hline ANGPT2 & $-2.1 \pm 0.3$ \\
KISS1 & $-2.5 \pm 0.4$ \\
ANTXR1 & $-2.8 \pm 0.4$ \\
CLDN11 & $-10.0 \pm 0.9$ \\
\hline
\end{tabular}

$\mathrm{p} \leq 0.05$ for all values.

qPCR confirmation of a subset of gene expression changes in HEMECs compared to HDMVECs

\section{Overexpression of the CTAG2 cancer/testis antigen in a panel of infantile hemangioma tumors}

In our microarray analysis, the cancer/testis antigen CTAG2 displayed the highest upregulation of mRNA expression in HEMECs compared to the HDMECs. This gene, whose function is completely unknown, has been shown to be significantly increased in several metastatic cancers, and is actively being researched as a target of immune therapy for aggressive cancers [ 23-29]. If CTAG2 is preferentially upregulated in infantile hemangiomas, it is possible that treatment of disfiguring or life threatening infantile hemangioma tumors could employ immune therapy against this antigen.

Furthermore, CTAG2 is reported to be a target for antigen-specific T-cells in patients with various metastatic tumors [ 29, 30]. A recent study has shown that nearly half of the patients with spontaneous CTAG2-specific CD4(+) T cell responses had circulating CTAG2-specific antibodies that recognized epitopes located in the C-terminal portion of CTAG2 [ 30]. As involution of infantile hemangiomas is believed to be due in part to an immune mediated attack on the tumor itself [ 4], it is possible that T-cell targeting of the overexpressed CTAG2 protein could contribute to this process. We confirmed our microarray data at the protein level by performing immunohistochemistry on a panel of 16 paraffin embedded infantile hemangioma tumors representing both the proliferating and involuting stages of the disease and 4 normal neonatal dermal tissues. A limited amount of CTAG2 expression was observed in the normal dermal tissues (a few nerve cells and bundles present staining, whereas the fibroblasts and collagen fibers are negative), and despite this gene being coined a "cancer/testis specific antigen", analysis of publically available microarray datasets suggests this gene is expressed at a low level across a large number of tissues ( http://www.biogps.org) and it has been reported in the literature to be expressed in the placenta and ovary [31]. In proliferating tumors (composed of densely proliferating endothelial cells), we observed intense CTAG2 staining in the endothelial cells for all sections analyzed (Figure 2). In contrast, involuting tumors (marked by substantial adipocyte deposits - a characteristic of the later stages in the development of this tumor [ 32]) exhibited significantly reduced levels of CTAG2 staining. As Calicchio et al. did not detect significant differences in CTAG2 expression between microdissected endothelial cells from proliferating and involuting infantile hemangiomas and the staining intensity of individual blood vessels appears relatively constant between proliferating and involuting hemangiomas, we suspect that the reduced CTAG2 staining in involuting tumors is most likely due to reductions in tumor vascular density but not changes in gene transcription.

\section{Figure 2}




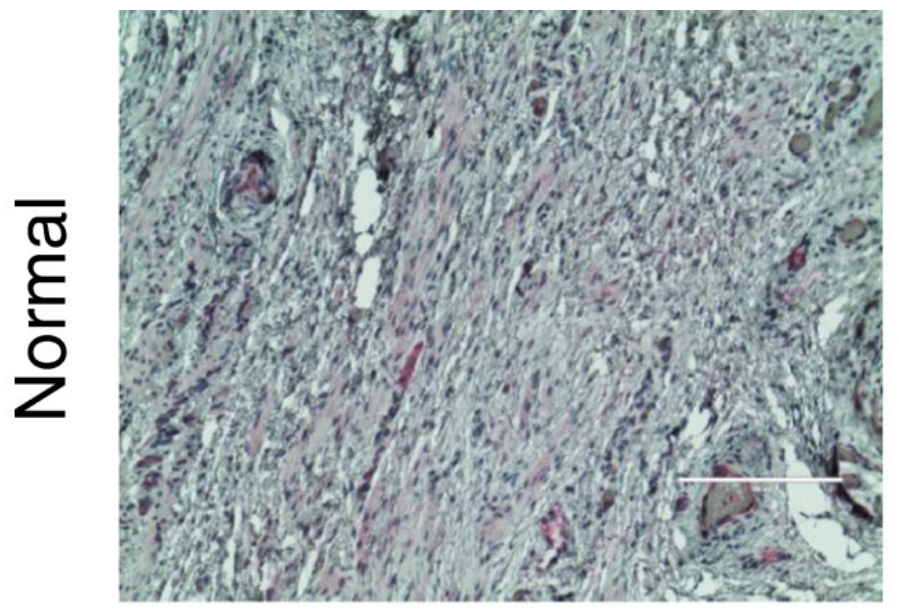

Neonatal Foreskin
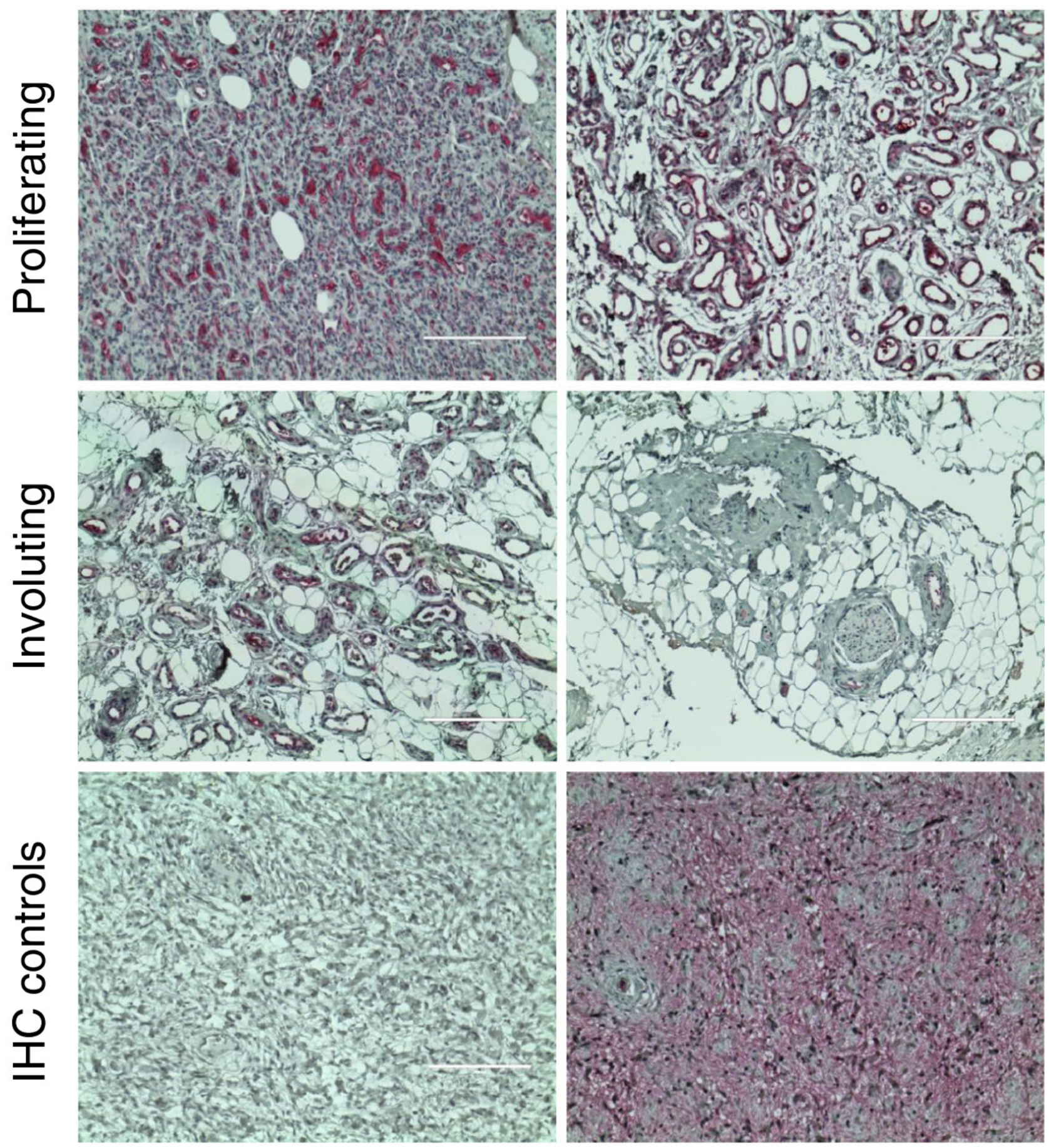

Negative Control

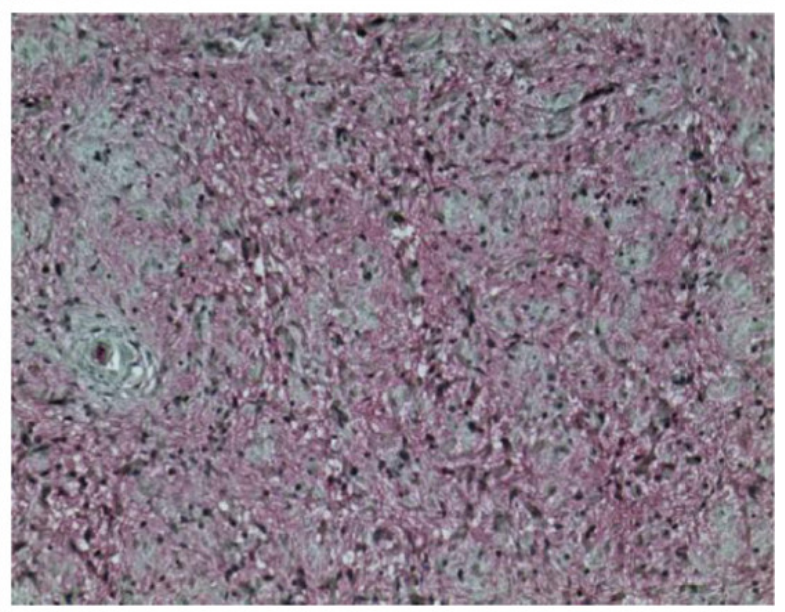

\section{Positive Control}


tissues as well as normal neonatal foreskin tissues were cut from paraffin blocks, incubated with antibodies against CTAG2, and detected using alkaline phosphatase staining ( red ). Immunohistochemistry ( IHC ) controls included incubations without CTAG2 antibody ( negative control) and with CTAG2 antibody ( positive control) in thin sections from metastatic breast cancer. All images were obtained at 100X total magnification.

\section{Conclusion}

Our data indicate that global transcriptional expression patterns are markedly unique between pure cultures of HDMVECs and HEMECs with major alterations in cell cycle, adhesion, and arachidonic acid metabolism genes. Though considered benign, HEMECs showed surprising aberrant regulation in the expression of several genes involved in tumor progression. Our finding that CTAG2 is highly expressed in infantile hemangiomas may lead to the development of immune-mediated therapies against infantile hemangiomas.

\section{Declarations}

\section{Authors' original submitted files for images}

Below are the links to the authors' original submitted files for images.

Authors' original file for figure 1

Authors' original file for figure 2

\section{Competing interests}

The authors declare that they have no competing interests.

\section{Authors' contributions}

JMS Performed proliferation and migration assays, confocal microscopy, and qPCR RKR Cultured the HDMVEC and HIHECs and prepared samples for microarray analysis. CA Generated and analyzed tables of statistically significant genes, performed bioinformatics analysis to identify key gene networks. DD Performed histology and immunohistochemistry. VK Provided human tissues and expertise in histological and immunohistochemistry analysis. DCM Performed microarray analysis and bioinformatics analysisc. BAB Wrote manuscript, analyzed data, coordinated project.

\section{References}

1. North PE, Waner M, Mizeracki A, Mihm MC. GLUT1: a newly discovered immunohistochemical marker for juvenile hemangiomas. Hum Pathol. 2000;31:11-22.

View Article Google Scholar

2. Mihm MC Jr Nelson JS Hypothesis: the metastatic niche theory can elucidate infantile hemangioma development J Cutan Pathol 201037 Suppl 183 873177757 10.1111/j.1600-0560.2010.01521.x

3. Barnes CM Huang S Kaipainen A Sanoudou D Chen EJ Eichler GS Guo Y Yu Y Ingber DE Mulliken JB Beggs AH Folkman J Fishman SJ Evidence by molecular profiling for a placental origin of infantile hemangioma Proc Natl Acad Sci USA 200510219097 191021323205 10.1073/pnas.0509579102

4. Ritter MR, Moreno SK, Dorrell MI, Rubens J, Ney J, Friedlander DF, Bergman J, Cunningham BB, Eichenfield L, Reinisch J, Cohen S, Veccione T, Holmes R, Friedlander SF, Friedlander M. Identifying potential regulators of infantile hemangioma progression through large-scale expression analysis: a possible role for the immune system and indoleamine 2,3 dioxygenase (IDO) during involution. Lymphat Res Biol. 2003;1:291-299. View Article Google Scholar

5. Calicchio ML Collins T Kozakewich HP Identification of signaling systems in proliferating and involuting phase infantile hemangiomas by genome-wide transcriptional profiling Am J Pathol 20091741638 16492671253 10.2353/ajpath.2009.080517

6. Boye E Yu Y Paranya G Mulliken JB Olsen BR Bischoff J Clonality and altered behavior of endothelial cells from hemangiomas J Clin Invest 2001107745752208946 10.1172/JCI11432

7. Stiles J Amaya C Pham R Rowntree RK Lacaze M Mulne A Bischoff J Kokta V Boucheron LE Mitchell DC Bryan BA Propranolol treatment of infantile hemangioma endothelial cells: A molecular analysis Exp Ther Med 201245946043501380 
8. Khan ZA Melero-Martin JM Wu X Paruchuri S Boscolo E Mulliken JB Bischoff J Endothelial progenitor cells from infantile hemangioma and umbilical cord blood display unique cellular responses to endostatin Blood 20061089159211895853 10.1182/blood-2006-03-006478

9. Pfister SL Gauthier KM Campbell WB Vascular pharmacology of epoxyeicosatrienoic acids Adv Pharmacol 20106027593373307 10.1016/B978-0-12-385061-4.00002-7

10. Fagiani E, Christofori G. Angiopoietins in angiogenesis. Cancer Lett. 2013;328:18-26. View Article Google Scholar

11. Chaudhary A St Croix B Selective blockade of tumor angiogenesis Cell Cycle 201211225322593383587 $10.4161 / \mathrm{cc} .20374$

12. Silini A Ghilardi C Figini S Sangalli F Fruscio R Dahse R Pedley RB Giavazzi R Bani M Regulator of Gprotein signaling 5 (RGS5) protein: a novel marker of cancer vasculature elicited and sustained by the tumor's proangiogenic microenvironment Cell Mol Life Sci 2012691167 11783299962 10.1007/s00018011-0862-8

13. Garcia-Pedrero JM, Kiskinis E, Parker MG, Belandia B. The SWI/SNF chromatin remodeling subunit BAF57 is a critical regulator of estrogen receptor function in breast cancer cells. J Biol Chem. 2006;281:22656-22664. View Article Google Scholar

14. Lethe B, Lucas S, Michaux L, De Smet C, Godelaine D, Serrano A, De Plaen E, Boon T. LAGE-1, a new gene with tumor specificity. Int J Cancer. 1998;76:903-908.

View Article Google Scholar

15. Wessells H Sullivan CJ Tsubota Y Engel KL Kim B Olson NE Thorner D Chitaley K Transcriptional profiling of human cavernosal endothelial cells reveals distinctive cell adhesion phenotype and role for claudin 11 in vascular barrier function Physiol Genomics 2009391001082765067

10.1152/physiolgenomics.90354.2008

16. Cho SG Yi Z Pang X Yi T Wang Y Luo J Wu Z Li D Liu M Kisspeptin-10, a KISS1-derived decapeptide, inhibits tumor angiogenesis by suppressing Sp1-mediated VEGF expression and FAK/Rho GTPase activation Cancer Res 200969706270703242001 10.1158/0008-5472.CAN-09-0476

17. Mitchell DC, Stafford LJ, Li D, Bar-Eli M, Liu M. Transcriptional regulation of KiSS-1 gene expression in metastatic melanoma by specificity protein-1 and its coactivator DRIP-130. Oncogene. 2007;26:1739-1747. View Article Google Scholar

18. Jinnin M Medici D Park L Limaye N Liu Y Boscolo E Bischoff J Vikkula M Boye E Olsen BR Suppressed NFAT-dependent VEGFR1 expression and constitutive VEGFR2 signaling in infantile hemangioma Nat Med 200814123612462593632 10.1038/nm.1877

19. Yu Y Varughese J Brown LF Mulliken JB Bischoff J Increased Tie2 expression, enhanced response to angiopoietin-1, and dysregulated angiopoietin-2 expression in hemangioma-derived endothelial cells Am J Pathol 2001159227122801850579 10.1016/S0002-9440(10)63077-5

20. London NR Whitehead KJ Li DY Endogenous endothelial cell signaling systems maintain vascular stability Angiogenesis 200912149 1582698036 10.1007/s10456-009-9130-z

21. Ramaesh T, Logie JJ, Roseweir AK, Millar RP, Walker BR, Hadoke PW, Reynolds RM. Kisspeptin-10 inhibits angiogenesis in human placental vessels ex vivo and endothelial cells in vitro. Endocrinology. 2010;151:5927-5934. View Article Google Scholar

22. Mitchell DC, Abdelrahim M, Weng J, Stafford LJ, Safe S, Bar-Eli M, Liu M. Regulation of KiSS-1 metastasis suppressor gene expression in breast cancer cells by direct interaction of transcription factors activator protein-2alpha and specificity protein-1. J Biol Chem. 2006;281:51-58.

View Article Google Scholar

23. Odunsi K, Jungbluth AA, Stockert E, Qian F, Gnjatic S, Tammela J, Intengan M, Beck A, Keitz B, Santiago D, Williamson B, Scanlan MJ, Ritter G, Chen YT, Driscoll D, Sood A, Lele S, Old LJ. NY-ESO-1 and LAGE-1 cancer-testis antigens are potential targets for immunotherapy in epithelial ovarian cancer. Cancer Res. 2003;63:6076-6083.

View Article Google Scholar 
24. Zeng G, Aldridge ME, Wang Y, Pantuck AJ, Wang AY, Liu YX, Han Y, Yuan YH, Robbins PF, Dubinett SM, deKernion JB, Belldegrun AS. Dominant B cell epitope from NY-ESO-1 recognized by sera from a wide spectrum of cancer patients: implications as a potential biomarker. Int J Cancer. 2005;114:268-273.

View Article Google Scholar

25. Kan T, Yamasaki S, Kondo K, Teratani N, Kawabe A, Kaganoi J, Meltzer SJ, Imamura M, Shimada Y. A new specific gene expression in squamous cell carcinoma of the esophagus detected using representational difference analysis and cDNA microarray. Oncology. 2006;70:25-33.

View Article Google Scholar

26. Shao Y, Sun ZY, Sun SW, Zhao Y, Sin WY, Yuan YH, Simpson AJ, Old LJ, Sang XT, Mao YL, Xie Y, Huang JF, Zhao HT. Identification and expression analysis of novel LAGE-1 alleles with single nucleotide polymorphisms in cancer patients. J Cancer Res Clin Oncol. 2008;134:495-502.

View Article Google Scholar

27. Andrade VC Vettore AL Felix RS Almeida MS Carvalho F Oliveira JS Chauffaille ML Andriolo A Caballero OL Zago MA Colleoni GW Prognostic impact of cancer/testis antigen expression in advanced stage multiple myeloma patients Cancer Immun 2008822935785

28. Wang XY, Chen HS, Luo S, Zhang HH, Fei R, Cai J. Comparisons for detecting NY-ESO-1 mRNA expression levels in hepatocellular carcinoma tissues. Oncol Rep. 2009;21:713-719.

View Article Google Scholar

29. Pollack SM Li Y Blaisdell MJ Farrar EA Chou J Hoch BL Loggers ET Rodler E Eary JF Conrad EU 3rd Jones RL Yee C NYESO-1/LAGE-1s and PRAME are targets for antigen specific T cells in chondrosarcoma following treatment with 5-Aza-2-deoxycitabine PLoS One 20127 e321653288075 10.1371/journal.pone.0032165

30. Kudela P Sun Z Fourcade J Janjic B Kirkwood JM Maillere B Zarour HM Epitope hierarchy of spontaneous CD4+ T cell responses to LAGE-1 J Immunol 20111863123223901358 10.4049/jimmunol.1001989

31. Kalejs M Erenpreisa J Cancer/testis antigens and gametogenesis: a review and "brain-storming" session Cancer Cell Int 200554552320 10.1186/1475-2867-5-4

32. Kleiman A, Keats EC, Chan NG, Khan ZA. Evolution of hemangioma endothelium. Exp Mol Pathol. 2012;93:264-272.

View Article Google Scholar 